\title{
EL VÍNCULO SOCIAL EN HIJO DE LADRÓN: SU INSCRIPCIÓN NACIONAL Y LA CUESTIÓN DE LA MIRADA ${ }^{1}$
}

\author{
Ignacio Álvarez \\ Universidad de Chile \\ Santiago, Chile \\ ignacioalvarez@uchile.cl
}

RESUMEN / ABSTRACT

El artículo describe Hijo de ladrón (1951) de Manuel Rojas como una novela de mejoramiento en la que un individuo aislado, Aniceto Hevia, logra vincularse con una comunidad de semejantes. Ese vínculo se caracteriza como esencialmente masculino, creado y no natural, basado en el trabajo y la resistencia a la adversidad. A partir de su contexto de producción y de su contexto de lectura, asimismo, proponemos que puede leerse como una alegoría nacional para el medio siglo chileno. Luego se estudia la cuestión de la mirada como experiencia afectiva fundante de esa comunidad. Ella ofrece una instancia fundamental de reconocimiento entre los miembros de la comunidad imaginada, que sería previa a su articulación política. Finalmente, se discuten brevemente ambos aspectos a la luz de la obra de Rojas y de otras formaciones alegóricas.

Palabras clave: Hijo de ladrón, Manuel Rojas, alegoría nacional, literatura chilena.

1 La segunda parte de este artículo, en una primera versión, fue presentada como ponencia: "El ojo y el corazón: la mirada y los afectos masculinos en Hijo de ladrón". XXI Congreso de la Sociedad Chilena de Estudios Literarios SOCHEL 2018: Figuras de lo común: formas y disensos en los estudios literarios (lenguas, cuerpos, sentidos y escrituras). Valparaíso, Pontificia Universidad Católica de Valparaíso, 6 al 9 de noviembre de 2018. Este artículo forma parte del proyecto FONDECYT 1140984, "El realismo narrativo como una idea fuera de lugar. Chile 1850-1990", del que el autor es investigador responsable. 


\section{THE SOCIAL BOND IN HIJO DE LADRÓN: \\ ITS NATIONAL INSCRIPTION AND THE GAZE ISSUE}

This article describes Hijo de ladrón as an improvement novel in which an isolated individual, Aniceto Hevia, can bond with a community of peers. This bond is characterized as essentially masculine, created and not natural, based on work and resistance to adversity. From its context of production and reading, we also propose that the novel can be interpreted as a national allegory of the middle Chilean $20^{\text {th }}$ Century. Then we describe the gaze as a founding affective experience in that community. It is a fundamental instance of recognition among the members of the imagined community, which would be prior to its political articulation. Finally, both aspects are briefly discussed in the light of Rojas's other works and other allegorical formations of Chilean literature.

KEYWORDS: Hijo de ladrón, Manuel Rojas, national allegory, Chilean literature.

Recepción: 31/01/2021

Aprobación: 01/03/2021

El hombre libre trata, pues, de ligarse a los demás hombres por la amistad.

Baruch Spinoza

Si hubiera que hacer un resumen elemental del argumento de Hijo de ladrón (1951), la novela más conocida y acaso la más importante de Manuel Rojas, no sería totalmente incorrecto decir que, en última instancia, el relato cuenta la historia de un joven que, luego de algunos tanteos y pasos en falso, logra integrarse a una comunidad de semejantes, logra construir un vínculo social.

Los hitos que articulan esa lectura serían dos. El inicio, que nos presenta a Aniceto Hevia, protagonista y narrador, delante de la cárcel de Valparaíso, por fin libre tras cumplir una injusta condena. Ha sido acusado de asaltar una joyería -"una joyería cuya existencia y situación ignoraba e ignoro aún” (Hijo de ladrón 17)- en el contexto de un motín ocurrido en esa ciudad a causa del precio del tranvía ${ }^{2}$. Es un sujeto aislado en su individualidad, alguien que no

\footnotetext{
Sobre este motín anota Manuel Rojas en su Antología autobiográfica: "El motín que se describe ocurrió en Valparaíso, en 1914 o principios de 1915, no recuerdo exactamente, y lo que ahí le sucede a Aniceto Hevia es exactamente lo que me ocurrió a mí, con la diferencia de que yo no enfermé; después de doce días de detención fui puesto en libertad" (374). El investigador Jorge Guerra ha establecido los datos que presentamos a continuación: los hechos ocurrieron entre el 28 de noviembre y el 4 diciembre 1914, motivados por el alza en el precio
} 
tiene personas a quienes recurrir o lugares adonde ir: "no existía, en aquella ciudad llena de gente y de poderosos comercios, un lugar, uno solo, hacia el cual dirigir mis pasos en busca de alguien que me ofreciera una silla, un vaso de agua, un amistoso apretón de manos o siquiera una palmadita en los hombros" (Hijo de ladrón 127). Todo cambia en el final: Aniceto se integra al pequeño grupo laboral y vital que forman el Filósofo Alfonso Echeverría y Cristián Ardiles, quienes sobreviven recolectando piezas de metal en la caleta El Membrillo, primero, y más tarde como una cuadrilla de pintores de brocha gorda en el litoral central de Chile. Las últimas líneas de la novela están dedicadas justamente a una escena de articulación social; Cristián, que ha sido renuente a integrarse a algún grupo, decide finalmente hacerse parte de este:

\section{- ¡Espérenme!}

Era un grito ronco, como de desgarramiento.

Nos detuvimos.

Cristián avanzó hacia nosotros.

Cuando se nos juntó reanudamos la marcha $(359)^{3}$.

Esta historia de mejoramiento no es solo el eje más elemental sino también el más estable de la novela, un eje que al comienzo es difícil de reconocer debido al denso montaje de los episodios, pero que luego va abriéndose como el horizonte de sentido del relato. Varios asedios analíticos han ido caracterizando la clase de vínculo que une a esta pequeña sociedad. Para

de los tranvías. E1 2 de diciembre el malestar explota en disturbios callejeros, y cerca de la una de la mañana se produce un enfrentamiento con la policía montada. El periódico La Unión de Valparaíso registra entre los detenidos en el barrio Almendral a Manuel Rojas Sepúlveda, y entre los heridos al guardián Gabriel Pérez, por pedrada en la espalda, algo que coincide con el relato de Aniceto en el capítulo V de la Segunda Parte de la novela (ver Hijo de ladrón 133, nota 3).

La adhesión de Cristián a la pequeña sociedad que forma con Echeverría y con Aniceto es frágil, pues su historia personal lo hace renuente a la vida en común: "Cristián tenía, por supuesto, una dureza, tal vez otra dureza, pero una que había hecho que resistiera años y años de cachetadas, puntapiés, calabozos, piojos, hambre, bubones, frío y soledad, una dureza que al mismo tiempo le impedía soportar la conmiseración o la dádiva, que no quería ni la una ni la otra y que tampoco le permitía o le habría permitido agradecer cualquiera de las dos" (Rojas, La oscura 35). Como se relata en La oscura vida radiante, termina dedicado a pequeños robos, y muere de un balazo en la boca cuando lo descubren dentro de una casa (36). 
José Promis es el fundamento que define la obra de Rojas como proyecto; cuando Aniceto Hevia pasa de un estado de aislamiento a uno de vinculación social está descubriendo "la causa profunda que explica el mecanismo de su existencia" (Promis 81). Trabajos como los de Grínor Rojo y Berta López Morales apuntan al sustrato ideológico de la sociedad que inaugura ese breve núcleo, su frontal oposición al sistema hegemónico o al orden burgués, una cuestión que está obviamente relacionada con la formación anarquista de Manuel Rojas. El modo en que se concibe la oposición al orden burgués define las calificaciones que Rojo y López Morales ofrecen a la novela: contraBildungsroman en el primer caso, novela de aprendizaje antiburguesa en el segundo ${ }^{4}$.

Este trabajo quiere proponer que, además de las lecturas anteriores, es posible inscribir la comunidad que inaugura Hijo de ladrón en una dimensión nacional. La operación tiene alguna dificultad, sin embargo. A diferencia de lo que ocurre en La oscura vida radiante (1970), la última novela de la tetralogía de Aniceto Hevia, en Hijo de ladrón el protagonista no se inserta explícitamente en la historia de Chile y, es más, suele definirse como argentino y renegar de la idea misma de nación ${ }^{5}$. Intentaré mostrar que Hijo de ladrón propone una renovación del pacto nacional: rechaza el nacionalismo decimonónico y ofrece una representación renovada de la comunidad imaginada, verdaderamente democratizadora e inclusiva. En segundo término, mostraré que el imaginario nacional de Rojas busca como fundamento remoto una experiencia concreta, una vivencia que lo hace posible: el encuentro amoroso de las miradas.

\section{EL VÍNCULO SOCIAL Y SU INSCRIPCIÓN NACIONAL}

Para describir la textura política del vínculo social que propone Hijo de ladrón, a mi juicio, hay que retroceder a sus primeros cuentos. Estudiando

\footnotetext{
4 Ver Rojo 186 y López Morales 313-317.

5 Sobre la inscripción nacional de La oscura vida radiante ver Álvarez, Novela y nación 122-128. En Hijo de ladrón el cuestionamiento burocrático de la nacionalidad aparece, por ejemplo, aquí: "Bueno, yo nací en Buenos Aires, pero eso no tenía valor alguno; lo valioso era el certificado; nunca me sirvió de nada el decirlo y las personas a quienes lo dije no demostraron en sus rostros de funcionarios entusiasmo ni simpatía alguna; faltaba el certificado; y los peores eran mis compatriotas: además de serles indiferente que fuera natural de Buenos Aires, no lo creían, pidiéndome, para creerlo, un certificado" (Hijo de ladrón 23).
} 
"Laguna", el primero de todos, Jaime Concha observa que la comunidad que allí se forma, tal como la de Aniceto, el Filósofo y Cristián, es esencialmente masculina y se organiza en torno a la experiencia del trabajo. Llama a ese vínculo "hombría", y describe su contenido del siguiente modo:

La hombría es quizás fundamentalmente eso, el poder enfrentar los golpes duros y las malas rachas como parte de lo que el destino depara a cada uno, entendiendo que hay una inevitable repartición de bienes y de males y que esa repartición es siempre impredecible, casi siempre injusta. Hombría: el hombre de cara a la adversidad (Concha, "Los primeros cuentos" 209).

En Hijo de ladrón también se puede reconocer el trabajo y la adversidad como rasgo aglutinante de la sociedad a la que se integra Aniceto. La invitación que Echeverría le hace es, precisamente, que recolecte con ellos los pedazos metálicos que se encuentran en la playa y que luego venderán, por un valor que apenas les permite subsistir, a un comerciante aragonés. Hay en este arreglo una dimensión no digamos estoica, porque no lo es, pero sí heroica en un sentido hegeliano, es decir, relativa el heroísmo del esclavo que prefiere padecer la vida a ejercer la violencia ${ }^{6}$. Ese heroísmo de los perdedores, esa forma de hombría se expresa justo después del encuentro, cuando el Filósofo pregunta a Aniceto por el tamaño de su mala racha, de su adversidad: “- - Lo persigue el león? Me preguntaba si tenía hambre y si me sentía acorralado. Aquello era tan evidente que me pareció inútil contestarle" (Hijo de ladrón 276). El león que lleva a Aniceto hacia la playa, el mismo que alguna vez correteó a Cristián y a Echeverría, los junta ahora en la labor, los iguala o, dicho en el idioma de Benedict Anderson, los vuelve una comunidad horizontal ${ }^{7}$.

\footnotetext{
Utilizo la lectura que Fredric Jameson hace de la dialéctica hegeliana del amo y el esclavo: "Dos iguales luchan por el reconocimiento del otro: uno está dispuesto a sacrificar la vida por ese valor supremo; el otro, un heroico cobarde en su amor desmedido al cuerpo y al mundo material - un amor brechtiano, schweykiano-, se entrega para asegurar la continuidad de la vida" (Jameson, "La literatura" 192).

Intento dialogar con la famosa definición de Benedict Anderson, que en Comunidades imaginadas describe la nación como "una comunidad política imaginada como eminentemente limitada y soberana" (23). Debo agregar una observación muy pertinente de Francisca C. Soto y Carlos Pavez, quienes identifican los momentos de construcción comunitaria en Lanchas en la bahía con la experiencia del tiempo presente en su duración. Se trata de una cuestión extensible a Hijo de ladrón sin problemas. Soto y Pavez identifican estos momentos con el
} 
Esta pequeña sociedad, en un principio reunión precaria de sujetos que apenas sobreviven, va adquiriendo progresivamente cierta densidad política. Ella aparece, por ejemplo, cuando discuten su modo de producción:

- Sin querer — dijo El Filósofo, una vez que estuvimos en la calle-, sin querer y en contra de su voluntad, lo he incorporado a la razón social Filósofo-Cristián.

-No entiendo - le dije.

—Sí —explicó—; junté su metal con el nuestro y ahora no sé cuánto es el suyo.

En respuesta me encogí de hombros.

-No pelearemos por el reparto.

Mostró los siete pesos, que apretaba en su mano larga y poco limpia, y dijo

-Y, para colmo, nos tocó un número difícil: siete. ¿Cuánto es siete dividido entre tres? A ver cómo ando para las matemáticas superiores: dos pesos para cada uno son seis pesos; queda uno, entre tres, treinta centavos; dos pesos treinta para cada uno y sobran diez cobres. Lo declararemos capital de reserva. Volvamos donde está Cristián (288).

Ironía calculada de la organización capitalista, en la comunidad de Aniceto no hay acumulación originaria, no se compite sino que se comparte equitativamente, tan equitativamente que hasta el cálculo preciso de la ganancia individual se vuelve ridículo y el pequeño excedente producido se conserva como ahorro común. Es un capitalismo llevado al absurdo, quizá, y por eso el Filósofo lo describe con un poco de sorna, o bien el capitalismo como podría y debería ser, uno en donde nadie se pelea por el reparto.

¿De qué manera puede inscribirse este vínculo social, descrito hasta aquí en sus rasgos más gruesos, como una alegoría nacional? En dos direcciones, a mi juicio: en tanto representación de su contexto, es decir, desde su producción, y como lectura a posteriori, es decir, desde su recepción.

En cuanto al polo de la producción del texto, conviene recordar la situación temporal de Hijo de ladrón. Es cierto, se enfoca en contar hechos ocurridos entre 1914 y 1915 (no exclusivamente, claro, pero allí está su foco), pero lo hace más o menos explícitamente desde una gran distancia temporal, una distancia cuyo terminus ad quem podríamos poner en 1951, el año de

tiempo homogéneo y vacío que Benedict Anderson propone como temporalidad propia de lo nacional (6). 
su publicación. Como se ha descrito varias veces, es un Aniceto ya mayor, integrado socialmente, maduro y letrado el que narra los hechos de un Aniceto joven, marginalizado y abierto a la experiencia (Álvarez, Novela y nación 103). Estas dos fechas están, además, en dos momentos históricos muy distintos del siglo XX chileno. El año 1915 -algo parecido se puede decir de 1920, e incluso de 1938- representa el estertor final de lo que Armando de Ramón ha llamado el "proyecto histórico de la oligarquía chilena", cuya versión de la nacionalidad, en forma de alegoría, puede leerse en Martín Rivas, de Alberto Blest Gana, tal como ha sido demostrado varias veces ${ }^{8}$. El año 1951, en cambio, se encuentra en la mitad del "proyecto histórico de las clases medias", es decir, un período de pacto entre las clases medias, la antigua oligarquía y el mundo popular, un pacto imperfecto y siempre tenso, por cierto, pero en donde existe un enorme crecimiento del Estado -el propio Manuel Rojas es funcionario de la Universidad de Chile- un gran impulso al desarrollo industrial y un proceso progresivo de democratización social ${ }^{9}$.

Esta disyunción temporal, me parece, también se reproduce en la lectura. La novela representa al mismo tiempo el Chile de 1915 (el del enunciado) y el Chile de 1951 (el de la enunciación), aunque de distintas maneras y construyendo distintos referentes. La crítica al nacionalismo de papel, a la nacionalidad de mero pasaporte, me parece, es una crítica al pacto oligárquico, que solo nominalmente incluía a los sujetos del mundo popular ${ }^{10}$. La nueva

8 Esta periodización del siglo XX aparece en la Historia de Chile de Armando de Ramón. El período 1810-1920 representaría el "proyecto histórico de la oligarquía chilena", y el trecho que va de 1920-1973 el "proyecto de las clases medias". Sobre el primero, cabe señalar que consistió fundamentalmente en la expansión territorial y la explotación de la riqueza material de ese territorio, con vistas a mantener la hegemonía de la clase oligárquica (De Ramón 66). Para la lectura de Martín Rivas (1862) de Alberto Blest Gana como alegoría clásica de la sociedad decimonónica ver Jaime Concha, "Martín Rivas o la formación del burgués". Leer a contraluz. Estudios sobre narrativa chilena. De Blest Gana a Varas y Bolaño.

De Ramón tiene una lectura muy optimista de este momento: “en el período 19381973 existió en el país una verdadera república donde las libertades, pero al mismo tiempo el respeto a los derechos de las personas, pasaron a ser una realidad sentida y ejercida por todos los sectores del país" (119). Cabe advertir que este juicio ha sido rebatido, desde posturas liberales, porque el desarrollo del Estado ha implicado una progresiva burocratización y limitación de las libertades individuales; desde posturas más radicales, porque el pacto que articulan las clases medias no es suficientemente democratizador y transformador.

10 Por ejemplo: “Aparece la autoridad: a ver los papeles. ¿Chileno? ¿Argentino? Muéstreme su libreta de enrolamiento, muéstreme su pasaporte, muéstreme su equipaje; por poco te piden que les muestres otra cosa. Y si vas sucio y rotoso, porque te ha ido mal en el 
sociedad a la que Aniceto se integra, la que es verdaderamente horizontal e interclasista, la que se funda en el trabajo y el saber soportar la adversidad, es decir, la que se articula en torno a un hacer en común, puede ser leída perfectamente como una versión idealizada y utópica del nuevo pacto nacional; como la representación posible y anhelada del proyecto que está en pleno despliegue mientras Rojas escribe. Se trata, además, de un proyecto del cual el propio Rojas forma parte, como lo demuestra su trayectoria biográfica, que lo llevó desde los extramuros de la sociedad chilena hasta su incorporación plena, como veremos en seguida, al campo literario nacional ${ }^{11}$.

La recepción de Hijo de ladrón, por otro lado, es en parte el relato de su apropiación nacional. Es cierto, la incorporación de Rojas al campo cultural chileno se puede rastrear mucho antes de la novela, cuando él mismo entendía que, aunque sus cuentos buscaban distanciarse de él, tenían varias semejanzas con el criollismo imperante ${ }^{12}$. En 1957, sin embargo, al recibir el Premio Nacional de Literatura, esa apropiación queda de alguna forma sellada. En su documentado y reciente estudio sobre el Premio Nacional como institución cultural, Pablo Faúndez Morán describe con cierto detalle el sentido que tuvo concedérselo a Manuel Rojas, y concluye que el jurado -formado por Juan Gómez Millas, rector de la Universidad de Chile, Carlos Préndez Saldías en representación del Ministerio de Educación y Ricardo Latcham a nombre de la Sociedad Chilena de Escritores- justifica el galardón con criterio fundamentalmente literario (ponderando la calidad que los propios escritores le reconocen dentro y fuera del país), pero también aludiendo a

trabajo o porque te da la gana ir rotoso y sucio, es mucho peor. Si no caes en gracia te llevarán al retén y te tendrán ahí dos horas o dos días o una quincena" (Hijo de ladrón 122).

11 La idea de que los textos representan sus contextos de producción en la enunciación, independientemente de los objetos de la representación del enunciado, aparece excelentemente argumentada en la noción de inconsciente político de Fredric Jameson. Ver Documentos de cultura 62-67. Allí también se presenta convincentemente, a mi juicio, la idea de que todos los textos narrativos son legibles como alegorías de sus contextos, uno de los cuales es la identidad nacional. Quiero esquivar, mediante este giro, la idea de alegoría nacional que el mismo Jameson ha defendido en su famoso ensayo "La literatura del Tercer Mundo en la era del capitalismo multinacional" (170-172). Para una relectura de la noción de alegoría nacional en Jameson, ver Álvarez, “Todos contra Jameson” 13.

12 En su ensayo "Acerca de la literatura chilena", de 1930, y hablando en tercera persona de sí mismo, da cuenta de este posicionamiento: "Citemos algunos escritores actuales y veamos sobre qué escriben o sobre qué han escrito. Mariano Latorre, costumbres campesinas; Marta Brunet, lo mismo; Luis Durand, ídem; Santiván, actualmente costumbres campesinas; Maluenda, Escenas de la vida campesina; Manuel Rojas, rotos y campesinos" (69). 
su representatividad, a su contenido vernáculo (97). Yo quisiera subrayar, por mi parte, que la decisión del jurado se apoya muy principalmente en Hijo de ladrón, y ello no solo porque es una novela de gran calidad, como sabemos, sino porque a la fecha no había más volúmenes publicados de lo que en el futuro llegaría a ser la tetralogía de Aniceto Hevia: Mejor que el vino solo sería publicada al año siguiente, en 1958, Sombras contra el muro aparecería en 1964 y La oscura vida radiante en $1970^{13}$. Esto quiere decir que no nos equivocamos demasiado si pensamos que Hijo de ladrón fue leída en su momento en un marco preponderantemente nacional y, por tanto, que la nueva sociedad que postula en su desenlace podía entenderse sin problemas como una alegoría de orden nacional.

¿Y cuál es la importancia, finalmente, de poder inscribir Hijo de ladrón como una alegoría nacional? Creo que ese valor se hace evidente cuando lo comparamos con la estructura que alegorizó la comunidad nacional durante el siglo XIX. Como sabemos, en novelas como Martín Rivas -y de manera invertida o indirecta en narraciones problemáticas como Casa grande y Juana Lucero-, la oligarquía propuso figurar su deseo de nación a través de una pareja heterosexual cuyo erotismo propone un vínculo natural o naturalizado para la nación; su fertilidad produce nuevos ciudadanos para la patria y, en virtud de la dependencia sanguínea del linaje, no puede sino excluir de la ciudadanía a los que no pertenecen a su clase ${ }^{14}$. La novela de Manuel Rojas elabora su alegoría de una manera muy distinta: ya no será el deseo heterosexual sino el afecto homosocial su fundamento, tampoco será una comunidad natural sino un moderno acuerdo basado en el reconocimiento mutuo, la productividad no se medirá en ciudadanos sino en aquello que pueda crear el trabajo. Su sistema de exclusiones no se basará en la clase social, sin embargo, sino en la construcción genérico sexual. La nación de Rojas es una nación de varones, y esa es su principal e insoslayable ceguera.

13 Cito la parte del fallo que me interesa: Manuel Rojas "se ha hecho acreedor no solo del aprecio de la crítica nacional sino de la más amplia aceptación de los entendidos y el favor del público internacional, como lo revelan las sucesivas ediciones de una de sus más sobresalientes novelas, Hijo de ladrón, que ha merecido la versión a diferentes lenguas extranjeras y la acogida de los más exigentes públicos de América y Europa" (citado en Faúndez Morán 97).

14 Solo reproduzco el análisis general de Doris Sommer para las ficciones fundacionales del siglo XX. Ver Sommer 5-6. 


\section{LA CUESTIÓN DE LA MIRADA}

El apartado anterior se hace cargo del vínculo que Aniceto conquista en la novela desde una perspectiva más o menos abstracta -la nación-y progresiva, en cuanto se relaciona estrechamente con el curso modernizador de Chile; ese vínculo, como vimos, surge de la experiencia del trabajo y del enfrentarse juntamente a la adversidad. La novela, sin embargo, registra como base de ese vínculo comunitario una experiencia más inmediata y presente, anterior incluso al intercambio entre sus miembros. Es lo que quiero describir en este apartado, pues se trata de algo que podríamos considerar como el primer germen de lo social, algo que se da en la dimensión sutil de los afectos y que tiene como vehículo la mirada.

La reflexión sobre la mirada es sorprendentemente antigua en la obra de Manuel Rojas. Aparece por primera vez en uno de sus cuentos menos conocidos, pero más estremecedores: se trata de "Poco sueldo", publicado por primera vez en 1929 en el diario La Nación. Allí se cuenta la historia de Laureano González, un obrero electricista que vive, al filo de lo posible, con un salario mísero que debe bastar para su familia y para su severa adicción al vino. Un día cualquiera el administrador de la empresa consigue verlo por primera vez y, sobrecogido, decide subirle el sueldo. El aumento lo mata, sin embargo: ahora puede tomar más allá de lo que su cuerpo resiste. Copio aquí la escena de esa primera inscripción de la mirada en Rojas:

El administrador da una mirada al obrero. Es la primera vez que lo mira detenidamente, a fondo. No tiene costumbre de mirar con detención a los trabajadores de la empresa. Los mira bien nada más que al tomarlos, para ver si son sanos, fuertes, si denotan hábitos de trabajo. Una vez colocados no los mira sino a la cara y rápidamente, al mandarlos o al saludarlos. Ignora cómo viven. No tiene tiempo de informarse. Pero esa mañana mira al hombre que tiene delante como se debe mirar a los hombres, de arriba abajo, para saber de ellos no solo lo que dicen o piensan, sino también lo que viven y lo que sienten. El examen le produce angustia; aquello no es un hombre, es un estropajo. Nunca ha visto tanta pobreza y tanto abandono ("Poco sueldo" 333 ).

Además de lo que ya conocemos -el trabajo y la sociabilidad masculina, la hombría de que hablaba Concha-el narrador de Rojas precisa que existe un buen modo de mirar y un modo malo de hacerlo, es decir, que la mirada puede 
describirse desde una perspectiva ética. Por otro lado, también puede hablarse del mirar como de un arte, un saber hacer, una práctica, una habilidad que permite penetrar aquello que se mira para conocerlo. Finalmente, aparece la cuestión del afecto como afectación: en su recorrido interior la mirada logra tocar íntimamente al hombre observado y conectarse con él en unos términos que no son ni racionales ni estamentales sino emotivos y existenciales.

"Poco sueldo" se publica en 1929, como apunté antes. Hijo de ladrón, en donde se retoma largamente el tema de la mirada, apareció en 1951 y, si hacemos caso al propio Rojas, fue elaborado lentamente durante buena parte de la década del cuarenta ${ }^{15}$. Esto significa que no se trata de un motivo circunstancial o caprichoso en su obra; corresponde a una figura que medita por más de veinte años. Cuando reaparece en Hijo de ladrón, como veremos, lo hace de una forma mucho más elaborada.

Volvamos al encuentro con Cristián y el Filósofo, en el capítulo tercero de la Tercera Parte de la novela. Lo precede una reflexión sobre la soledad que siente Aniceto al salir de la cárcel, una reflexión que primero se hace en clave familiar: "Yo no tenía, en cambio, a nadie: la familia de mi madre parecía haber desaparecido" (269). Luego se repite, traducida ahora al idioma del mirar: "No tenía en Chile hacia quién volver la cara; no era nada para nadie, nadie me esperaba o me conocía en alguna parte y debía aceptar o rechazar lo que me cayera en suerte" (269-270, el énfasis es mío): no tenía hacia quién volver la cara, es decir, nadie a quién mirar y nadie que lo mire.

Cuando se produce, inmediatamente después, el cruce efectivo de las miradas, ello ocurre en tres tiempos que propongo comentar por separado. Quien primero los distingue es Aniceto, a lo lejos, pero luego los observa con detención:

Seguí mirando; los dos hombres daban la impresión de que eran nacidos en aquella playa llena de cabezas de sierra, tripas de pescada, aletas de azulejos, trozos de tentáculos de jibia y tal cual esqueleto de pájaro marino; hedionda, además, a aceite de bacalao y decorada

15 En "Algo sobre Hijo de ladrón", Rojas indica que comenzó a escribir la novela cerca de 1936 o 1937 cuando, habiendo enviudado recientemente, debió complementar los ingresos que obtenía como Director de Prensas de la Universidad de Chile con la corrección de traducciones y la venta de cartillas en el Hipódromo. En 1940 publicó en la revista Babel un primer fragmento de ese proyecto, llamado "Ensayo de la mañana". Recién en 1950 dio por terminado el libro $(366-7 ; 376)$. 
por graves alcatraces. No eran, sin embargo, pescadores, que era fácil reconocer por sus sombreros sin color y sin forma determinada, sus pies descalzos, sus inverosímiles chalecos -siempre más grandes que cualesquiera otros y que nunca parecen ni son propios, como los de los tonys-y sus numerosos suéteres, no. Sus vestimentas, por lo demás, no decían nada acerca de sus posibles oficios, ya que una chaqueta verdosa y lustrada, con el forro y la entretela viéndose tanto por encima como por debajo, con unos bolsillos que más eran desgarraduras y unos pantalones con flecos y agujeros por todas partes, no podían dar indicios sobre sus sistemas de ganarse la vida. De una cosa, sin embargo, se podía estar seguro: sus rentas no llegarían a incomodarlos por lo copiosas (270-271).

Aunque no lo sabe todavía, Aniceto mira a dos hombres que se encuentran en la mitad de su jornada laboral, con todo lo informal y precaria que ella sea. Su mirar, que por cierto es un buen mirar, hace surgir a los hombres de entre la materia que los rodea. Quiere comprenderlos y se los explica por su circunstancia, por su contexto, por el mundo que los contiene, un mundo que se le abalanza a través del olfato. La comprensión objetiva y material que Aniceto intenta, por otro lado, no tiene como finalidad la clasificación cosificadora: comprender a un hombre no es clasificarlo, y la buena vista debe conformarse a veces con dejar zonas en la penumbra. Pronto sabrá Aniceto cuál es el oficio de estos hombres surgidos de la entraña de la playa.

El segundo tiempo es una reacción y corresponde a la mirada que Cristián echa sobre Aniceto:

Por su parte, también me miraron, uno primero, el otro después, una mirada de inspección, y el primero en hacerlo fue el que marchaba por el lado que daba hacia la calle y cuya mirada me traspasó como un estoque: mirada de gaviota salteadora, lanzada desde la superficie del ojo, no desde el cerebro, y estuve seguro de que mi imagen no llegó, en esa primera mirada, más allá de un milímetro de su sistema visual exterior. Era para él un simple reflejo luminoso, una sensación desprovista de cualquier significado subjetivo. No sacó nada de mí: me miró como el pájaro o el pez miran al pez o al pájaro, no como a algo que también está vivo, que se alimenta de lo mismo que él se alimenta y que puede ser amigo o enemigo, pero que siempre es, hasta que no se demuestre lo contrario, enemigo. Era quizá la mirada de los hombres de las alcantarillas, llena de luz, pero superficial, que solo ve y siente la sangre, la fuerza, el ímpetu, el propósito inmediato (271). 
La imagen de la skúa o gaviota salteadora es harto decidora y seguramente tenía alguna importancia descriptiva para Rojas. En un cuento del mismo año 1951, "Mares libres", utiliza a esta misma ave marina como protagonista, y la pinta por medio de un brochazo rotundo: "es la más desalmada: ningún pájaro puede pescar a su vista ni el más miserable de los peces sin correr el riesgo de que ella se lo arrebate a picotazos" (375). Me parece más importante, sin embargo, establecer la "física" de este mirar de Cristián a Aniceto. Es una mirada material, en primer lugar, una mirada que punza, que traspasa y penetra, aunque ahora no con intención exploratoria o cognoscitiva sino con deseo de agredir y hacer daño. La mirada de Cristián, vuelta arma, ejerce violencia antes de mirar incluso, antes de reconocer a quien está mirando, y ello porque es una mirada que no ve, que no distingue.

La posibilidad de entender esta mirada en términos más o menos mecánicos recuerda la descripción que hacía Baruch Spinoza de los afectos, que define del siguiente modo: "por afecto entiendo las afecciones del cuerpo, con las que se aumenta o disminuye, ayuda o estorba la potencia del actuar del mismo cuerpo, y al mismo tiempo, las ideas de estas afecciones" (Ética $3,1)^{16}$. No quiero -ni tampoco podría- entrar en el detalle filosófico de este problema, que tanto se ha debatido durante los últimos y penúltimos años. Sin embargo, hay un par de datos que provienen de este campo que pueden ser especialmente útiles para entender la cuestión de la mirada en Rojas. El primero de ellos tiene que ver con el tratamiento de la dimensión interior del ser humano con unas leyes que son igualmente aplicables al mundo mecánico y exterior. El segundo tiene que ver con la cuestión de la variedad: "cuanto más apto es un cuerpo para hacer o padecer más cosas a la vez”, dice Spinoza, "más apta que las demás es su alma para percibir a la vez más cosas" (Ética 2, prop. 12, escolio).

16 Las relaciones entre Manuel Rojas y el pensamiento spinozista son todavía un terreno que debe ser explorado. Hay, sin embargo, algunas pistas que vale la pena recordar y que Susan Linker ha explicitado. En primer lugar, debe mencionarse la estrecha amistad entre Rojas y Enrique Espinoza, que no es otro que el editor y ensayista argentino Samuel Glusberg, cuya cercanía con las ideas de Spinoza era tan estrecha que escogió ese apellido como parte de su pseudónimo. En segundo término, es bien probable que el "Padre Espinoza" de "El hombre de la rosa" también esté inspirado en la figura de Baruch (Linker 23). En 1978, Enrique Espinoza publicó un breve libro llamado Spinoza, águila y paloma, de donde recogemos dos aforismos: el que está en el epígrafe de este trabajo y este otro, la única mención a los afectos que hay en ese breve libro: "Un afecto que es una pasión deja de serlo tan pronto como nos formamos de él una idea clara y distinta. Un afecto que es una pasión, es una idea clara y distinta" (78). 
Si comparamos la mirada de Cristián y la de Aniceto en tanto afecciones, podremos ver algunas diferencias significativas. Como si fuera una varilla de dos extremos, la mirada de Cristián es afilada y peligrosa en el extremo que ofrece a Aniceto, y en cambio es débil y roma en el extremo que está más cerca de él: "estuve seguro de que mi imagen no llegó, en esa primera mirada, más allá de un milímetro de su sistema visual exterior" (271). Lo contrario puede decirse de la mirada de Aniceto, que más que varilla parece una escoba, pues aunque hasta las más sutiles diferencias del mundo se le hacen ostensibles, aunque ve mucho, es incapaz de hacer daño. La monótona mirada de Cristián no ve sino enemigos, no ve sino solo una cosa, no ve. Por lo mismo, ataca. La mirada de Aniceto ve todo o ve mucho. Por lo mismo, espera. Si los sentidos son parte del cuerpo, es dable pensar, siguiendo a Spinoza, que el alma de Aniceto esté más abierta y más cercana a la sabiduría.

El tercer tiempo lo ocupa el Filósofo Echeverría mirando a Aniceto:

Desvió [Cristián] la mirada y pasó de largo y le tocó entonces al otro hombre mirarme, una mirada que fue la recompensa de la otra, porque este, sí, este me miró como una persona debe mirar a otra, reconociéndola y apreciándola como tal desde el principio; una mirada también llena de luz, pero de una luz que venía desde más allá del simple ojo. Sonrió al mismo tiempo; una sonrisa que no se debía a nada, ya que por allí no se veía algo que pudiera hacer sonreír; tal vez una sonrisa que le sobraba y de las cuales tendría muchas. Una mirada me traspasó, la otra me reconoció (271-272).

Reconocemos de inmediato la ética y el arte de mirar que Rojas había estrenado en "Poco sueldo". Como bien sabemos, el Filósofo no está mirando a Aniceto motu proprio; su mirada es una respuesta a la mirada primera del muchacho, una reacción, un afecto en sentido spinoziano. La sonrisa de Echeverría confirma que la mirada de Aniceto "aumenta o ayuda la potencia del actuar", que la mutua perturbación corporal y mental será fecunda en el futuro. Esa sonrisa es, en efecto, la evaluación de la mirada primera de Aniceto, y proviene del interior del Filósofo, un interior que no es romo ni ciego, un interior en donde hay mucho más para dar, como sabremos en adelante.

Esta descripción del arte de mirar en Hijo de ladrón nos permite postular la existencia de una instancia que, siendo necesaria para la conformación de la comunidad, le antecede largamente. Dicha instancia puede quizá describirse con el idioma del afecto, algo anterior a la convivencia, anterior a cualquier trabajo en conjunto, anterior a la hombría y por cierto anterior a cualquier 
pacto político. Su anterioridad, muy probablemente, implica que cualquier renovación de la convivencia política requiera el contacto amoroso de los rostros. Los miembros de la comunidad que Hijo de ladrón propone reconocen y son reconocidos aun cuando no se conozcan, son rostros que miran a otros rostros y que se dejan mirar.

\section{DISCUTIR UN IMAGINARIO NACIONAL}

Mirarse los unos a los otros, trabajar juntos, soportar el sufrimiento y repartir entre todos los hombres el fruto del trabajo: estos son los rasgos que, de acuerdo a lo que he intentado exponer, definen el vínculo social que propone Hijo de ladrón. Si hacemos caso a la lectura alegórica, no se trata efectivamente de acciones cara a cara, cuestión imposible para la nación en tanto comunidad imaginada; podemos decodificarlas, al interior de un orden fuertemente patriarcal, como el reconocimiento de las diferencias, la solidaridad común, la postulación de un orden político más justo.

Creo que la pequeña sociedad que representan Aniceto, el Filósofo y Cristián puede leerse con una triple valencia: como una crítica frontal a las formas de sociabilidad decimonónica que estaban desmoronándose durante la década del diez del siglo XX; como una expresión de la nueva comunidad que lentamente va modernizándose y democratizándose, pero también como una crítica a ese mismo presente de la enunciación de la novela, el medio siglo chileno, que al mismo tiempo, de una forma u otra, hace posible a Manuel Rojas como escritor.

Respecto al imaginario decimonónico, vale la pena apuntar el reemplazo del afecto erótico por el reconocimiento homosocial: a lo inevitable y natural que tiene la alegoría amorosa, se le opone la construcción de un vínculo moderno, creado, basado en la experiencia común. Resulta interesante que veamos resurgir la alegoría decimonónica en algunas novelas posteriores a Hijo de ladrón, siempre de la mano de un esquema y una perspectiva social oligárquicos, aun cuando tengan ahora la marca de una decadencia incontrarrestable: no otro es el fundamento compositivo de Casa de campo (1981) de José Donoso.

La adhesión de Rojas a la marcha de la modernidad chilena del siglo XX será cada vez menos evidente a partir de la década del 50, por otro lado, e irá progresivamente predominando una mirada crítica de ese proceso, algo que 
veremos en la cada vez más concentrada atención que sus novelas pondrán en el período 1915-1920, y muy especialmente en su última novela, $L a$ oscura vida radiante ${ }^{17}$.

La violencia, tema espinudo que Rojas abordará más profundamente en Sombras contra el muro, también asoma su problemática cabeza en este imaginario nacional. Ni la nueva comunidad ni ninguna puede evitarlo, parece decirnos el narrador de Hijo de ladrón a través de la figura de Cristián Ardiles y su modo de mirar. En las subjetividades crónicamente marginalizadas se apoza un resto de agresión que precede al pacto social y por tanto a los ciudadanos. Debemos aprender a convivir con ella.

\section{BIBLIOGRAFÍA}

Álvarez, Ignacio. Novela y nación en el siglo XX chileno. Ficción literaria e identidad. Santiago: Ediciones de la Universidad Alberto Hurtado, 2009.

"Todos contra Jameson: los alcances de la alegoría nacional y su valor para la lectura de dos novelas chilenas recientes". Ponencia presentada en el XIII Congreso internacional de la Sociedad Chilena de Estudios Literarios. 30 de septiembre de 2004. Consultado en https://www.academia.edu/1746207/Todos_contra_Jameson_los_alcances_de_la alegor\%CC3\%ADa_nacional_y_su_valor_para_la_lectura_de dos_novelas_chilenas_recientes

Anderson, Benedict. Comunidades imaginadas. Reflexiones sobre el origen y difusión del nacioanlismo. Trad. Eduardo L. Suárez. México: Fondo de Cultura Económica, 1993.

Concha, Jaime. "Los primeros cuentos de Manuel Rojas". Eds. Naín Nómez y Emmanuel Tornés Reyes. Manuel Rojas. Estudios críticos. Santiago: Editorial Universidad de Santiago, 2005. 333-351.

"El otro tiempo perdido". Leer a contraluz. Estudios de narrativa chilena. De Blest Gana a Bolaño y Varas. Santiago: Ediciones Universidad Alberto Hurtado, 2011. 223-244.

De Ramón, Armando. Historia de Chile. Desde la invasión incaica hasta nuestros días (15002000). Santiago: Catalonia, 2003.

Espinoza, Enrique. Spinoza, águila y paloma. Buenos Aires: Babel, 1978.

Faúndez Morán, Pablo. El Premio Nacional de Literatura en Chile. La construcción de una importancia. Valparaíso: Ediciones Universitarias de Valparaíso, 2020.

17 Encuentra su lugar aquí la lectura de Jaime Concha: Rojas habría buscado en sus novelas recuperar un tiempo irremediablemente perdido, el tiempo de su esperanza utópica, continuamente mortificada por el desarrollo del siglo XX (Concha, "El otro tiempo" 239-244). 
Jameson, Fredric. Documentos de cultura, documentos de barbarie. La narrativa como acto socialmente simbólico. Trad. Tomás Segovia. Madrid: Visor, 1989. (Traducción de The Political Unconscious. Narrative as a Socially Symbolic Act).

"La literatura del Tercer Mundo en la era del capitalismo multinacional". 1986. Trad. Ignacio Álvarez. Revista de Humanidades 23 (2011): 163-193.

Linker, Susan M. "A Collision of Rationalism and Spiritualism in 'El hombre de la rosa' of Manuel Rojas: Decoding the Secret Signals”. Hispanic Review 68. 1 (2000): 21-36.

López Morales, Berta. "El aprendizaje de Aniceto Hevia”. Eds. Naín Nómez y Emmanuel Tornés Reyes. Manuel Rojas. Estudios críticos. Santiago: Editorial Universidad de Santiago, 2005. 287-317.

Promis, José. La novela chilena del último siglo. Santiago: La Noria, 1993.

Rojas, Manuel. “Algo sobre Hijo de ladrón”. Hijo de ladrón. Santiago: Tajamar, 2018. 361-390. “Acerca de la literatura chilena". 1930. De la poesía a la revolución. Santiago: Ercilla, 1938. 63-83. 375-385.

"Mares libres". Cuentos. Santiago: Ediciones Universidad Alberto Hurtado, 2016.

"Poco sueldo". Cuentos. Santiago: Ediciones Universidad Alberto Hurtado, 2016. 327-338.

Hijo de ladrón. Edición establecida por David Barrera, Diego Leiva, Alejandra Caballero e Ignacio Álvarez. Santiago: Tajamar, 2018.

La oscura vida radiante. Santiago: Lom, 2008.

Rojo, Grínor. "La contrabildungsroman de Manuel Rojas”. Las novelas de formación chilenas. Santiago: Sangría, 2014. 165-266.

Sommer, Doris. Ficciones fundacionales. Las novelas nacionales de América Latina. 1991. Trads. José Leandro Urbina y Ángela Pérez. Bogotá: Fondo de Cultura Económica, 2004.

Soto, Francisca C. y Carlos Pavez. "Lanchas en la bahía e Hijo de ladrón: temporalidad y comunidad". Manuscrito inédito, 2020.

Spinoza, Baruch. Ética demostrada según el orden geométrico. Ed. y trad. Atiliano Domínguez. Madrid: Trotta, 2005. 